\title{
ON STRONG APPROXIMATION BY MODIFIED MEYER-KÖNIG AND ZELLER OPERATORS
}

\author{
L. REMPULSKA AND M. SKORUPKA
}

\begin{abstract}
We introduce certain modified Meyer-König and Zeller operators $M_{n ; r}$ in the space of $r$-th times differentiable functions $f$ and we study strong differences $H_{n ; r}^{q}(f)$ for them.

This note is motivated by results on strong approximation connected with Fourier series ([7]).
\end{abstract}

\section{Introduction}

1.1. The Meyer-König and Zeller operators $M_{n}, n \in N=\{1,2, \ldots$,$\} ,$

$$
\begin{gathered}
M_{n}(f ; x):= \begin{cases}\sum_{k=0}^{\infty} p_{n k}(x) f\left(\frac{k}{n+k}\right) & \text { if } 0 \leq x<1, \\
f(1) & \text { if } x=1,\end{cases} \\
p_{n k}(x):=\left(\begin{array}{c}
n+k \\
k
\end{array}\right) x^{k}(1-x)^{n+1}, \quad k \in N_{0}=N \cup\{0\},
\end{gathered}
$$

associated with bounded functions $f: I=[0,1] \rightarrow R$, were introduced in [9].

Approximation properties of $M_{n}$ were given in many papers (e.g. [1, 3, 5, 8, 9].

In many papers (e.g. $[2,4,10])$ were introduced certain modifications of operators $M_{n}$ and were studied their properties in various functions spaces.

Let $C_{I}$ be the space of all functions $f: I \rightarrow R$, continuous on $I$ with the norm

$$
\|f\|=\sup \{|f(x)|: x \in I\} .
$$

It is known $([1,3,8])$ that $M_{n}, n \in N$, is a positive linear operator from the space $C_{I}$ into $C_{I}$ and for every $f \in C_{I}$ we have

$$
\left\|M_{n}(f)\right\| \leq\|f\|, \quad n \in N,
$$

and

$$
\left\|M_{n}(f)-f\right\| \leq \frac{31}{27} \omega\left(f ; \frac{1}{\sqrt{n}}\right), \quad n \in N,
$$

Received September 20, 2004; revised December 07, 2004.

2000 Mathematics Subject Classification. 41A36.

Key words and phrases. Meyer-König and Zeller operator, linear operator, strong approximation. 
where $\omega(\cdot, \cdot)$ is the modulus of continuity of $f([11])$, i.e.

$$
\omega(f ; t)=\sup \{|f(x)-f(y)|: x, y \in I,|x-y| \leq t\}, \quad t \in I .
$$

From (5) immediatelly follows

$$
\lim _{n \rightarrow \infty}\left\|M_{n}(f)-f\right\|=0,
$$

for every $f \in C_{I}$.

Let $r \in N_{0}$ be a fixed number and let $C_{I}^{r}:=\left\{f \in C_{I}: f^{(r)} \in C_{I}\right\}$ and the norm in $C_{I}^{r}$ is defined by $(3)\left(C_{I}^{0} \equiv C_{I}\right)$.

It is known ([1]-[3]) that if $f \in C_{I}^{r}, r \geq 2$, then

$$
\left\|M_{n}(f)-f\right\|=O\left(\frac{1}{n}\right), \quad n \in N,
$$

and this result cannot be improved.

1.2. In this note we shall show that certain modification of the formula (1) improves the approximation order (7) for functions $f \in C_{I}^{r}, r \geq 2$.

We introduce the following.

Definition. Let $r \in N_{0}$ be a fixed number. For $f \in C_{I}^{r}$ and $n \in N$ we define the following modified Meyer-König and Zeller operators:

$$
M_{n}(f ; x):= \begin{cases}\sum_{k=0}^{\infty} p_{n k}(x) \sum_{j=0}^{r} \frac{f^{(j)}\left(\xi_{n k}\right)}{j !}\left(x-\xi_{n, k}\right)^{j} & \text { if } 0 \leq x<1, \\ f(1) & \text { if } x=1,\end{cases}
$$

where

$$
\xi_{n k}:=\frac{k}{n+k}, \quad n \in N, \quad k \in N_{0},
$$

and $p_{n k}(x)$ is defined by (2).

Obviously $M_{n ; 0}(f ; x) \equiv M_{n}(f ; x)$ for $f \in C_{I}^{0}, x \in I$ and $n \in N$.

From (1), (2), (8) and (9) it follows that

$$
M_{n ; r}(1 ; x)=\sum_{k=0}^{\infty} p_{n k}(x)=1 \text { for } x \in I, \quad n \in N, \quad r \in N_{0} .
$$

In Section 2 we shall prove that $M_{n ; r}$ is a linear operator from the space $C_{I}^{r}$ into $C_{I}$. The main approximation theorem will be given also in Section 2 .

In this paper we shall denote by $K_{i}(a, b), i \in N$, suitable positive constants depending only on indicated paramenters $a, b$. 
1.3. Let $r \in N_{0}$ and $q>0$ be fixed numbers. For $f \in C_{I}^{r}$ and $M_{n ; r}(f)$ we introduce strong differences with the power $q$ as follows:

$$
H_{n ; r}^{q}(f ; x):= \begin{cases}\left(\sum_{k=0}^{\infty} p_{n k}(x)\left|\sum_{j=0}^{r} \frac{f^{(j)}\left(\xi_{n k}\right)}{j !}\left(x-\xi_{n, k}\right)^{j}-f(x)\right|^{q}\right)^{\frac{1}{q}} & \text { if } x \in[0,1), \\ 0 & \text { if } x=1,\end{cases}
$$

In particular for $f \in C_{I}, n \in N$ and $q>0$ we have

$$
H_{n ; 0}^{q}(f ; x):= \begin{cases}\left(\sum_{k=0}^{\infty} p_{n k}(x)\left|f\left(\xi_{n k}\right)-f(x)\right|^{q}\right)^{\frac{1}{q}} & \text { if } 0 \leq x<1, \\ 0 & \text { if } x=1,\end{cases}
$$

The properties of $H_{n ; r}^{q}(f)$ will be given in Section 2.

\section{Lemmas and Theorem}

2.1. First we shall give auxiliary results.

Lemma 1. For every $s \in N$ there exists $K_{1}(s)=$ const. $>0$ such that

$$
M_{n ; 0}\left(|t-x|^{s} ; x\right) \equiv M_{n}\left(|t-x|^{s} ; x\right) \leq K_{1}(s) n^{-\frac{s}{2}},
$$

for all $x \in I$ and $n \in N$.

Proof. In [4] was given the following inequality

$$
M_{n}\left((t-x)^{2 s} ; x\right) \leq K_{2}(s) n^{-s} \text { for } x \in I \text { and } n, s \in N,
$$

where $K_{2}(s)$ is suitable positive constant dependent only on $s$.

Using the Hölder inequality to $M_{n}\left(|t-x|^{s} ; x\right)$ and by $(10)$ and the above result, we immediately obtain the desired inequality.

Now we shall prove analogue of the inequality (4).

Lemma 2. Let $n, r \in N$ be fixed numbers. Then $M_{n ; r}(f)$ is a linear operator from the space $C_{I}^{r}$ into $C_{I}$ and

$$
\left\|M_{n ; r}(f)\right\| \leq \sum_{j=0}^{r}\left\|f^{(j)}\right\|
$$

for every $f \in C_{I}^{r}$.

Proof. Let $f \in C_{I}^{r}$ with $r \in N$. By (2), (3) and (9) we have

$$
\left|\sum_{j=0}^{r} \frac{f^{(j)}\left(\xi_{n k}\right)}{j !}\left(x-\xi_{n k}\right)^{j}\right| \leq \sum_{j=0}^{r} \frac{\left\|f^{(j)}\right\|}{j !}\left|x-\xi_{n k}\right|^{j} \leq \sum_{j=0}^{r} \frac{\left\|f^{(j)}\right\|}{j !}
$$


for all $x \in I, k \in N_{0}$ and $n \in N$. From this and by (8) - (10) we deduce the continuity of $M_{n ; r}(f)$ on interval $[0,1)$ and

$$
\left|M_{n ; r}(f ; x)\right| \leq \sum_{j=0}^{r}\left\|f^{(j)}\right\| \quad \text { for } x \in[0,1), \quad n \in N .
$$

Now we shall prove the continuity of $M_{n ; r}(f)$ at $x=1$.

If $f \in C_{I}^{r}, r \in N$, then the functions $h_{j, s}(x)=x^{s} f^{(j)}(x), x \in I, j, s=0,1, \ldots, r$, belong to $C_{I}$ and by properties of operators $M_{n}$ given in Section 1 we have also $M_{n}\left(h_{j, s}\right) \in$ $C_{I}$ and $\lim _{x \rightarrow 1-} M_{n}\left(h_{j, s}(t) ; x\right)=h_{j, s}(1)$, i.e.

$$
\lim _{x \rightarrow 1-} M_{n}\left(t^{s} f^{(j)}(t) ; x\right)=f^{(j)}(1), \quad 0 \leq j, \quad s \leq r .
$$

From the above and by (8) and (1) we get

$$
M_{n ; r}(f ; x)=\sum_{j=0}^{r} \frac{1}{j !} \sum_{s=0}^{j}\left(\begin{array}{l}
j \\
s
\end{array}\right)(-1)^{s} x^{j-s} M_{n}\left(t^{s} f^{(j)}(t) ; x\right)
$$

for $x \in[0,1)$, which by (15) and the equality

$$
\sum_{s=0}^{j}\left(\begin{array}{l}
j \\
s
\end{array}\right)(-1)^{s}= \begin{cases}1 & \text { if } j=0 \\
0 & \text { if } j \in N\end{cases}
$$

implies that

$$
\lim _{x \rightarrow 1-} M_{n}(f ; x)=\sum_{j=0}^{r} \frac{f^{(j)}(1)}{j !} \sum_{s=0}^{j}\left(\begin{array}{l}
j \\
s
\end{array}\right)(-1)^{s}=f(1) .
$$

The formulas (8), (16) and (17) show that $M_{n ; r}$ with $n, r \in N$ is a linear operator from the space $C_{I}$ into $C_{I}$. Moreover from (8) and (14) immedialely follow (13).

Applying Lemma 1 and Lemma 2 we can prove the following.

Lemma 3. Let $r \in N_{0}$ be a fixed number. Then $H_{n ; r}^{q}(f ; x)$ is well-defined for every $f \in C_{I}^{r}, x \in I, n \in N$ and $q>0$. Moreover the formula (11) can be written in the form:

$$
H_{n ; r}^{q}(f ; x):= \begin{cases}\left(M_{n}\left(\left|\sum_{j=0}^{r} \frac{f^{(j)}(t)}{j !}(x-t)^{j}-f(x)\right|^{q} ; x\right)\right)^{\frac{1}{q}} & \text { if } x \in[0,1), \\ 0 & \text { if } x=1,\end{cases}
$$

By elementary calculations we obtain.

Lemma 4. Suppose that $f \in C_{I}^{r}$ with a fixed $r \in N_{0}$. Then for $x \in I$ and $n \in N$ we have

$$
\left|M_{n ; r}(f ; x)-f(x)\right| \leq H_{n ; r}^{1}(f ; x)
$$


and

$$
H_{n ; r}^{p}(f ; x) \leq H_{n ; r}^{q}(f ; x) \quad \text { if } 0<p<q<\infty
$$

Proof. The formulas (8)-(10) and (1) imply that

$$
\begin{aligned}
\left|M_{n ; r}(f ; x)-f(x)\right| & =\left|M_{n}\left(\sum_{j=0}^{r} \frac{f^{(j)}(t)}{j !}(x-t)^{j}-f(x) ; x\right)\right| \\
& \leq M_{n}\left(\left|\sum_{j=0}^{r} \frac{f^{(j)}(t)}{j !}(x-t)^{j}-f(x)\right| ; x\right)
\end{aligned}
$$

for $0 \leq x<1$ and $M_{n ; r}(f ; 1)-f(1)=0$, which by (18) yield (19).

Applying the Hölder inequality and (10), we get

$$
\left(M_{n}\left(|g(t)|^{p} ; x\right)\right)^{\frac{1}{p}} \leq\left(M_{n}\left(|g(t)|^{q} ; x\right)\right)^{\frac{1}{q}}, \quad x \in I, \quad n \in N,
$$

for every $g \in C_{I}$ and $0<p<q<\infty$. From (18) and (21) immediately follows (20).

2.2. Applying the above lemmas we shall prove the main theorem.

Theorem. Let $r \in N_{0}$ and $q>0$ be fixed numbers. Then there exists $K_{5}(q, r)=$ const. $>0$ such that for every $f \in C_{I}^{r}$ and $n \in N$ we have

$$
\left\|H_{n ; r}^{q}(f ; \cdot)\right\| \leq K_{5}(q, r) n^{-\frac{r}{2}} \omega\left(f^{(r)} ; \frac{1}{\sqrt{n}}\right) .
$$

Proof. First let $r \in N$ and $q \in N$. Analogously to [6] we apply the following modified Taylor formula of $f \in C_{I}^{r}$ at a fixed point $x_{0} \in I$ :

$$
\begin{aligned}
f(x)= & \sum_{j=0}^{r} \frac{f^{(j)}\left(x_{0}\right)}{j !}\left(x-x_{0}\right)^{j} \\
& +\frac{\left(x-x_{0}\right)^{r}}{(r-1) !} \int_{0}^{1}(1-t)^{r-1}\left(f^{(r)}\left(x_{0}+t\left(x-x_{0}\right)\right)-f^{(r)}\left(x_{0}\right)\right) d t, \quad x \in I .
\end{aligned}
$$

Setting $x_{0}=\frac{k}{n+k} \equiv \xi_{n k}$ and using (23) to (11), we can write

$$
H_{n ; r}^{q}(f ; x)=\left(\sum_{k=0}^{\infty} p_{n k}(x)\left|\frac{\left(x-\xi_{n k}\right)^{r}}{(r-1) !} I_{n, k, r}\right|^{q}\right)^{\frac{1}{q}} \quad \text { for } \quad x \in[0,1) \text {, }
$$

where

$$
I_{n, k, r}:=\int_{0}^{1}(1-t)^{r-1}\left(f^{(r)}\left(\xi_{n k}+t\left(x-\xi_{n k}\right)\right)-f^{(r)}\left(\xi_{n k}\right)\right) d t .
$$

By (6) and properties of modulus of continuity ([11]) we have

$$
\begin{aligned}
& \left|f^{(r)}\left(\xi_{n k}+t\left(x-\xi_{n k}\right)\right)-f^{(r)}\left(\xi_{n k}\right)\right| \leq \omega\left(f^{(r)} ; t\left|x-\xi_{n k}\right|\right) \\
\leq & \omega\left(f^{(r)} ;\left|x-\xi_{n k}\right|\right) \leq \omega\left(f^{(r)} ; \frac{1}{\sqrt{n}}\right)\left(\sqrt{n}\left|x-\xi_{n k}\right|+1\right),
\end{aligned}
$$


for $0 \leq t \leq 1$ and further

$$
\left|I_{n, k, r}\right| \leq \frac{1}{r} \omega\left(f^{(r)} ; \frac{1}{\sqrt{n}}\right)\left(\sqrt{n}\left|x-\xi_{n k}\right|+1\right) .
$$

From the above and by the Minkowski inequality and (1) we get

$$
\begin{aligned}
H_{n ; r}^{q}(f ; x) & \leq \frac{1}{r !} \omega\left(f^{(r)} ; \frac{1}{\sqrt{n}}\right)\left(\sum_{k=0}^{\infty} p_{n k}(x)\left(\sqrt{n}\left|x-\xi_{n k}\right|^{r+1}+\left|x-\xi_{n k}\right|^{r}\right)^{q}\right)^{\frac{1}{q}} \\
& \leq \frac{1}{r !} \omega\left(f^{(r)} ; \frac{1}{\sqrt{n}}\right)\left(\sqrt{n}\left(M_{n}\left(|t-x|^{(r+1) q} ; x\right)\right)^{\frac{1}{q}}+\left(M_{n}\left(|t-x|^{q r} ; x\right)\right)^{\frac{1}{q}}\right),
\end{aligned}
$$

for $x \in[0,1), n \in N$. Now applying Lemma 1 , we easily obtain

$$
H_{n ; r}^{q}(f ; x) \leq K_{6}(q, r) n^{-\frac{r}{2}} \omega\left(f^{(r)} ; \frac{1}{\sqrt{n}}\right),
$$

for $x \in[0,1)$ and $\in N$. From this and (11) and (3) follows (22) for $q \in N$ and $r \in N$.

If $r \in N$ and $0<q \notin N$, then $[q]+1$ belongs to $N$ and $q<[q]+1$ ([q] is the integral part of $q$ ). Applying (20) and (3), we get

$$
\left\|H_{n ; r}^{q}(f ; \cdot)\right\| \leq\left\|H_{n ; r}^{[q]+1}(f ; \cdot)\right\|, \quad n \in N,
$$

which by (22) for $\left\|H_{n ; r}^{[q]+1}(f ; \cdot)\right\|$ implies (22) for $r \in N$ and $0<q \notin N$.

b) If $r=0$ and $f \in C_{I}$, then by (12) and (6) we have

$$
H_{n ; 0}^{q}(f ; x):= \begin{cases}\left(M_{n}\left(|f(t)-f(x)|^{q} ; x\right)\right)^{\frac{1}{q}} & \text { if } 0 \leq x<1, \\ 0 & \text { if } x=1,\end{cases}
$$

and

$$
|f(t)-f(x)| \leq \omega(f ;|t-x|) \leq \omega\left(f ; \frac{1}{\sqrt{n}}\right)(\sqrt{n}|t-x|+1)
$$

for $t, x \in I$. Arguing as in the case $r \in N$ and using (25) to (24), we obtain (22) for $r=0$.

Thus the proof is completed.

2.3. Finaly we shall give some corollaries and remarks.

From imequalities (19) and (20) and by (3) we deduce that

$$
\left\|M_{n ; r}(f)-f\right\| \leq\left\|H_{n ; r}^{1}(f)\right\| \leq\left\|H_{n ; r}^{q}(f)\right\|, \quad n \in N,
$$

for every $f \in C_{I}, r \in N_{0}$, and $q>1$.

The inequality (26) shows that our theorem on the strong approximation (with the power $q \geq 1$ ) of $f \in C_{I}^{r}$ by $M_{n ; r}(f)$ implies the classical approximation theorem for them.

From Theorem and (26) we derive the following two corollaries. 
Corollary 1. For every $f \in C_{I}^{r}, r \in N_{0}$, and $q>0$ we have

$$
\lim _{n \rightarrow \infty} n^{\frac{r}{2}}\left\|H_{n ; r}^{q}(f)\right\|=0
$$

and

$$
\lim _{n \rightarrow \infty} n^{\frac{r}{2}}\left\|M_{n ; r}(f)-f\right\|=0 .
$$

Corollary 2. Suppose that $f \in C_{I}^{r}, r \in N_{0}$, and $f^{(r)} \in \operatorname{Lip} \alpha$ with $0<\alpha \leq 1$, i.e. $\omega\left(f^{(r)} ; t\right)=O\left(t^{\alpha}\right), t \in(0,1]$. Then

$$
\left\|H_{n ; r}^{q}(f)\right\|=O\left(n^{-\frac{r+\alpha}{2}}\right), \quad n \in N,
$$

for every fixed $q>0$. Consequently we have

$$
\left\|M_{n ; r}(f)-f\right\|=O\left(n^{-\frac{r+\alpha}{2}}\right), \quad n \in N
$$

Remark. The given theorem (also the above corollaries) shows that the order of strong approximation of $f \in C_{I}^{r}, r \in N$, by $M_{n ; r}(f)$ is better than for classical MeyerKönig and Zeller operators $M_{n}(f)$. Moreover, Theorem shows that the order of strong approximation of $f \in C_{I}^{r}$ by $M_{n ; r}(f)$ improves if $r$ increases.

The identical properties we deduce from (27), (28) and (5) for ordinary approximation of $f \in C_{I}^{r}$ by $M_{n ; r}, r \in N$.

\section{References}

[1] M. Becker and R. J. Nessel, A global approximation theorem for Meyer-König and Zeller operators, Math. Z. 160(1978), 195-206.

[2] W. Chen, On the integral type Meyer-König and Zeller operators, Approx. Theory and its Applic. 2(1986), 7-18.

[3] R. A. De Vore, The Approximation of Continuous Functions by Positive Linear Operators, Springer, new York, 1972.

[4] S. Guo, On the rate of convergence of integrated Meyer-König and Zeller operators for functions of bounded variation, J. Approx. Theory 56(1989), 245-255.

[5] V. Gupta, A note on Meyer-König and Zeller operators for functions of bounded variation, Approx. Theory and its Applic. 18(2002), 99-102.

[6] G. H. Kirov, A generalization of the Bernstein polynomials, Math. Balkanica 6(1992), $147-153$.

[7] L. Leindler, Strong Approximation by Fourier Series, Akad. Kiado, Budapest, 1985.

[8] A. Lupas and M. W. Müller, Approximation properties of the $M_{n}$-operators, Aequationes Math. 5(1970), 19-37.

[9] W. Meyer-König and K. Zeller, Bernsteiniche Potenzreihen, Studia Math. 19(1960), 89-94.

[10] M. W. Müller, $L_{p}$-approximation by the method of integral Meyer-König and Zeller operators, Studia Math. 63(1978), 81-88.

[11] A. F. Timan, Theory of Approximation of Functions of Real Variable, New York, 1963. 
Institute of Mathematics, Poznań University of Technology, ul. Piotrowo 3A, 60-965 Poznań, Poland.

E-mail: lrempuls@math.put.poznan.pl

Institute of Mathematics, Poznań University of Technology, ul. Piotrowo 3A, 60-965 Poznań, Poland.

E-mail: mariolas@math.put.poznan.pl 